Proyecciones Journal of Mathematics

Vol. 30, $\mathrm{N}^{o}$ 1, pp. 1-17, May 2011.

Universidad Católica del Norte

Antofagasta - Chile

\title{
Cycle connectivity in weighted graphs
}

\author{
SUNIL MATHEW \\ NATIONAL INSTITUTE OF TECHNOLOGY, INDIA \\ and \\ M. S. SUNITHA \\ NATIONAL INSTITUTE OF TECHNOLOGY, INDIA \\ Received: August 2010. Accepted : December 2010
}

\begin{abstract}
Some new connectivity concepts in weighted graphs are introduced in this article. The concepts of strong arc, partial cutnode, bridge and block are introduced. Also three different types of cycles namely locamin cycle, multimin cycle and strongest strong cycle are introduced. Partial blocks in weighted graphs are characterized using strongest paths. Also a set of necessary conditions for a weighted graph to be a partial block involving strong cycles and a sufficient condition for a weighted graph to be a partial block involving strongest strong cycles are obtained. A new connectivity parameter called cycle connectivity and a new type of weighted graphs called $\theta$ - weighted graphs are introduced and partial blocks in $\theta$ - weighted graphs are fully characterized.
\end{abstract}

AMS classification : 05C22, 05C38, $05 C 40$.

Keywords : Weighted graph, partial cutnode, partial bridge, strong cycle, cycle connectivity. 


\section{Introduction}

Weighted graph theory has numerous applications in various fields like clustering analysis, operations research, database theory, network analysis, information theory, etc. Connectivity concepts play a key role in applications related with graphs and weighted graphs. Several authors including Bondy and Fan [1, 2], Broersma, Zhang and Li [9], Mathew and Sunitha [6, 7, 8] introduced many connectivity concepts in weighted graphs following the works of Dirac[4] and Grotschel [5].

In this article we introduce some new connectivity concepts in weighted graphs. In a weighted graph model, for example, in information networks and electric circuits, the reduction of flow between pairs of nodes is more relevant and may frequently occur than the total disruption of the flow or the disconnection of the entire network [6]. This concept is our motivation. As weighted graphs are generalized structures of graphs, the concepts introduced in this article also generalizes the classic connectivity concepts.

A weighted graph $G$ is one in which every arc $e$ is assigned a nonnegative number $w(e)$, called the weight of $e$. The set of all the neighbors of a vertex $v$ in $G$ is denoted by $N_{G}(v)$ or simply $N(v)$, and its cardinality by $d_{G}(v)$ or $d(v)$ [3]. The weighted degree of $v$ is defined as $d_{G}^{w}(v)=\sum_{x \in N(v)} w(v x)$.

When no confusion occurs, we denote $d_{G}^{w}(v)$ by $d^{w}(v)$. The weight of a cycle is defined as the sum of the weights of its edges. An unweighted graph can be regarded as a weighted graph in which every edge $e$ is assigned weight $w(e)=1$. Thus, in an unweighted graph, $d^{w}(v)=d(v)$ for every vertex $v$, and the weight of a cycle is simply the length of the cycle. An optimal cycle is a cycle which has maximum weight[1].

\section{Strong Cycles}

In a weighted graph $G$, to each pair of nodes, we can associate a real number called strength of connectedness. It is evaluated using strengths of different paths joining the given pair of nodes. We have a set of new definitions which are given below.

Definition 1: [7] Let $G$ be a weighted graph. The strength of a path $P$ of $n$ edges $e_{i}$, for $1 \leq i \leq n$, denoted by $s(P)$, is equal to $s(P)=$ 
$\min _{1 \leq i \leq n}\left\{w\left(e_{i}\right)\right\}$.

Consequently the strength of a cycle $C$ in a weighted graph $G$ is the minimum of the weights of $\operatorname{arcs}$ in $C$.

Definition 2:[7] Let $G$ be a weighted graph. The strength of connectedness of a pair of nodes $u, v \in V(G)$, denoted by $C O N N_{G}(u, v)$ is defined as $\operatorname{CONN}_{G}(u, v)=\operatorname{Max}\{s(P): P$ is a $u-v$ path in $G\}$. If $u$ and $v$ are in different components of $G$, then $C O N N_{G}(u, v)=0$.

Example 1: Consider the following weighted graph $G(V, E)$.

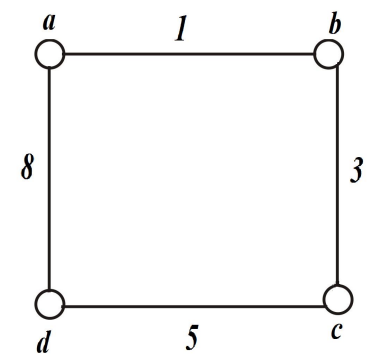

Figure 1 : Strength of connectedness

Here $C O N N_{G}(a, b)=3, C O N N_{G}(a, c)=5, C O N N_{G}(a, d)=8$, $C O N N_{G}(b, c)=3, C O N N_{G}(b, d)=3, C O N N_{G}(c, d)=5$.

Next we have an obvious result.

Proposition 1:[7] Let $G$ be a weighted graph and $H$, a weighted subgraph of $G$. Then for any pair of nodes $u, v \in G$, we have $C O N N_{H}(u, v) \leq$ $C O N N_{G}(u, v)$.

Definition 3:[7] A u-v path in a weighted graph $G$ is called a strongest $u-v$ path if $s(P)=C O N N_{G}(u, v)$.

Definition 4:[7] Let $G$ be a weighted graph. A node $w$ is called a partial cutnode (p-cutnode) of $G$ if there exists a pair of nodes $u, v$ in $G$ such that $u \neq v \neq w$ and $C O N N_{G-w}(u, v)<C O N N_{G}(u, v)$. A connected weighted graph having no p-cutnodes is called a partial block(p-block). 
Example 2: Let $G(V, E)$ be the following weighted graph.

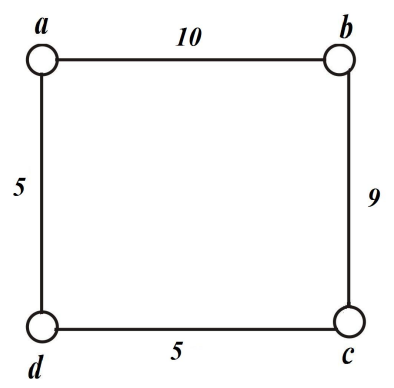

Figure 2: Weighted graph with a p-cutnode

Node $b$ is a partial cutnode since CON $N_{G-b}(a, c)=5<9=C O N N_{G}(a, c)$. Also note that the path $a b c$ is the unique strongest $a-c$ path in $G$.

Definition 5: [7] Let $G$ be a weighted graph. An arc $e=(u, v)$ is called a partial bridge (p-bridge) if $C O N N_{G-e}(u, v)<C O N N_{G}(u, v)$. A p-bridge is said to be a partial bond (p-bond) if $C O N N_{G-e}(x, y)<C O N N_{G}(x, y)$ with at least one of $x$ or $y$ different from both $u$ and $v$ and is said to be a partial cutbond(p-cutbond) if both $x$ and $y$ are different from $u$ and $v$.

Example 3: Consider the following weighted graph with four nodes.

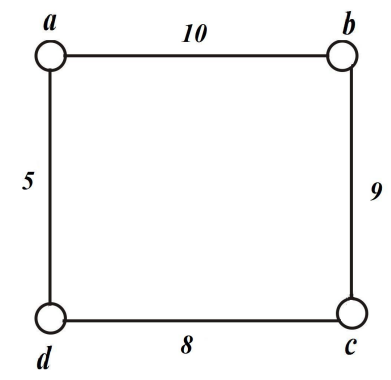

Figure 3 : Weighted graph with a p-cutbond

Here all arcs except arc $(a, d)$ are partial bonds. In particular arc $(b, c)$ is a partial cutbond since $C O N N_{G-(b, c)}(a, d)=5<8=C O N N_{G}(a, d)$. 
Definition 6: [7] Let $G$ be a weighted graph. Then an arc $e=(x, y) \in E$ is called $\alpha$ - strong if $C O N N_{G-e}(x, y)<w(e), \beta$ - strong if $C O N N_{G-e}(x, y)=$ $w(e)$ and $a \delta$ - arc if $C O N N_{G-e}>w(e) . A \delta$ - arc e is called a $\delta^{*}$ - arc if $e$ is not a weakest arc of $G$.

Clearly an $\operatorname{arc} e$ is strong if it is either $\alpha$ - strong or $\beta$ - strong. That is arc $(x, y)$ is strong if its weight is at least equal to the strength of connectedness between $x$ and $y$ in $G$. If $(x, y)$ is a strong arc, then $x$ and $y$ are said to be strong neighbors to each other.

Definition 7: [7] $A u-v$ path $P$ in $G$ is called a strong $u-v$ path if all arcs in $P$ are strong. In particular if all arcs of $P$ are $\alpha-$ strong, then $P$ is called an $\alpha$ - strong path and if all arcs of $P$ are $\beta$ - strong, then $P$ is called a $\beta$ - strong path.

Definition 8: [7] Let $G$ be a weighted graph and $C$, a cycle in $G . C$ is called a strong cycle if all arcs in $C$ are strong.

Example 4: Let $G(V, E)$ be a weighted graph with $V=\{a, b, c, d\}$ and $E=\left\{e_{1}=(a, b), e_{2}=(b, c), e_{3}=(c, d), e_{4}=(d, a), e_{5}=(a, c)\right\}$ with $w\left(e_{1}\right)=7, w\left(e_{2}\right)=8, w\left(e_{3}\right)=2, w\left(e_{4}\right)=2, w\left(e_{5}\right)=4$.

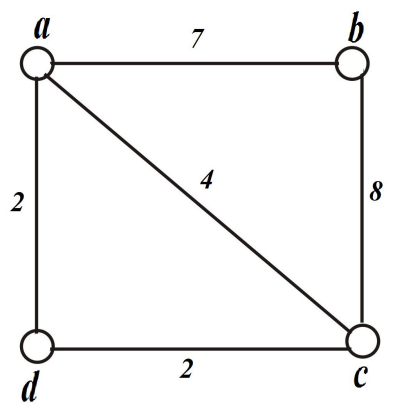

Figure $4: \alpha$-strong, $\beta$ - strong and $\delta$ - $\operatorname{arcs}$

Here, $(a, b)$ and $(b, c)$ are $\alpha$-strong, $(c, d)$ and $(d, a)$ are $\beta$ - strong and arc $(a, c)$ is a $\delta$-arc. Clearly arc $(a, c)$ is $\delta^{*}$ since it is not a weakest arc in $G$. Also $P_{1}=a b c$ is an $\alpha$-strong path, $P_{2}=c d a$ is a $\beta$-strong path. In $G, C_{1}=a b c d a$ is a strong cycle but $C_{2}=$ abca is not a strong cycle.

Theorem 1: A connected weighted graph $G$ is a partial block if and only if any two nodes $u, v \in V(G)$ such that $(u, v)$ is not $\alpha$ - strong are joined 
by two internally disjoint strongest paths.

Proof: Suppose that $G$ is a partial block. Let $u, v \in V(G)$ such that $(u, v)$ is not $\alpha$ - strong. To show that there exist two internally disjoint strongest $u-v$ paths. Suppose not. That is there exist exactly one internally disjoint strongest $u-v$ path in $G$. Since $(u, v)$ is not $\alpha$ - strong, length of all strongest $u-v$ paths must be at least two (Note that if $(u, v)$ is $\beta$-strong, then there exist two internally disjoint $u-v$ paths, which is not possible). Also for all strongest $u-v$ paths in $G$, there must be a node in common. Let $w$ be such a node in $G$. Then,

$C O N N_{G-w}(u, v)<C O N N_{G}(u, v)$, which contradicts the fact that $G$ has no p-cutnodes.

Conversely suppose that any two nodes of $G$ are joined by two internally disjoint strongest paths. Let $w$ be a node in $G$. For any pair of nodes, $x, y \in V(G)$ such that $x \neq y \neq w$, there always exist a strongest path not containing $w$. So $w$ cannot be a p-cutnode and the theorem is proved.

Theorem 2: Let $G$ be a connected weighted graph and let $x$ and $y$ be any two nodes in $G$. Then there exists a strong path from $x$ to $y$.

Proof: Suppose that $G$ is a connected weighted graph. Let $x$ and $y$ be any two nodes of $G$. If $\operatorname{arc}(x, y)$ is strong, there is nothing to prove. Otherwise, either $(x, y)$ is a $\delta$ - arc or there exist a path of length more than one from $x$ to $y$. In the first case we can find a path $P$ (say) such that $s(P)>w((x, y))$. In either case consider the path from $x$ to $y$ of length more than one. If some arc on this path is not strong, replace it by a path having more strength. This argument cannot be repeated arbitrary often; hence eventually we can find a path from $x$ to $y$ on which all the arcs are strong.

Theorem 3: If Gis a partial block then the following conditions hold and are equivalent.

(i)Every two nodes of $G$ lie on a common strong cycle.

(ii) Each node and a strong arc of $G$ lie on a common strong cycle.

(iii) Any two strong arcs of $G$ lie on a common strong cycle. 
(iv) For two given nodes and a strong arc in $G$ there exists a strong path joining the nodes containing the arc.

(v) for every three distinct nodes of $G$ there exist strong paths joining any two of them containing the third.

(vi) For every three nodes of $G$ there exist strong paths joining any two of them which does not contain the third.

\section{Proof:}

(i) Suppose that $G$ is a partial block. Let $u$ and $v$ be any two nodes in $G$ such that there exists a unique strong path between $u$ and $v$.

Now two cases arise.

(1) $(u, v)$ is a strong arc.

(2) $(u, v)$ is either a $\delta$-arc or there exist a $u-v$ path of length more than two in $G$.

Case 1: $(u, v)$ is a strong arc.

Since $(u, v)$ is not on any strong cycle, $(u, v)$ is an arc in every maximum spanning tree of $G$ and hence it is a partial-bridge. If $u$ is an end node in all Maximum Spanning Trees, then clearly $v$ is a p-cutnode in $G$ or vice versa contradicting our assumption that $G$ is a partial block. Now suppose that $u$ is an end node in some MST $T_{1}$ and $v$ is an end node in some MST $T_{2}$. Let $u^{\prime}$ be a strong neighbor of $u$ in $T_{2}$. Since $u$ is an end node and $v$ is an internal node in $T_{1}$, there exists a strong path $P$ in $T_{1}$ from $u$ to $u^{\prime}$ passing through $v$. The path $P$ together with the strong arc $\left(u, u^{\prime}\right)$ forms a strong cycle in $G$, a contradiction.

Case 2: Either $(u, v)$ is a $\delta$-arc or there exist a strong $u-v$ path of length more than two in $G$.

If $(u, v)$ is a $\delta$-arc, then there exists a strong path between $u$ and $v$. Since there is a unique strong $(u, v)$ path $P$ in $G, P$ belongs to all maximum spanning trees. Thus all internal nodes in $P$ are internal nodes in all the maximum spanning trees and hence all of them are partial cut nodes in $G$, contradiction to the assumption that $G$ is a partial block. 
$(i) \Rightarrow(i i)$ Suppose that every two nodes of $G$ lie on a common strong cycle. To prove that a given node and a strong arc lie on a common strong cycle. Let $u$ be a node and $v w$ be an arc in $G$. Let $C$ be a strong cycle containing $u$ and $v$. If $w$ is a neighbor of $v$ in $C$, then there is nothing to prove. Now suppose that $w$ is not a neighbor of $v$ in $C$. Let $C_{1}$ be a strong cycle containing $u$ and $w$. Let $P_{1}$ and $P_{2}$ be the strong $u-v$ paths in $C$ and $P_{1}^{\prime}$ and $P_{2}^{\prime}$ the strong $u-w$ paths in $C_{1}$.

Let $x_{1}$ be the node at which $P_{1}^{\prime}$ leaves $P_{1}$. Then clearly $u \ldots\left(P_{1}\right) \ldots x_{1} \ldots\left(P_{1}^{\prime}\right) \ldots w v \ldots\left(P_{2}\right) u$ is a strong cycle containing $u$ and $v w$. If $x=u$ then $\left.u . .\left(P_{1}^{\prime}\right)\right) . . w v . .\left(P_{2}\right) . . u$ is the required cycle. If $x_{1}=v$, let $x_{2}$ be the node at which $P_{2}^{\prime}$ leaves $P_{2}$. Then $u . .\left(P_{1}\right) . . v w \ldots .\left(P_{2}^{\prime}\right) . . x_{2} . .\left(P_{2}\right) u$ is the required strong cycle. If $x_{2}=u$ then $u . .\left(P_{2}^{\prime}\right) . . w v . .\left(P_{1}\right) . . u$ is the required strong cycle. Since $P_{1}$ and $P_{2}$ are internally disjoint both $x_{1}$ and $x_{2}$ cannot be the node $v$.

(ii) $\Rightarrow$ (iii) Suppose that each node and strong arc lies on a common strong cycle. To prove any two strong arcs lie on a common strong cycle. Let $u v$ and $x y$ be two strong $\operatorname{arcs}$ of $G$. Let $P_{1}$ and $P_{2}$ be two internally disjoint strong paths between $v$ and $x$ and $Q_{1}$ and $Q_{2}$ be two internally disjoint strong paths between $u$ and $y$. If $P_{1}, P_{2}, Q_{1}$ and $Q_{2}$ are internally disjoint, then $u v \ldots\left(P_{1}\right) \ldots x y \ldots\left(Q_{2}\right) \ldots u$ is a strong cycle containing $u v$ and $x y$. If $Q_{1}$ and $Q_{2}$ intersect $P_{1}$ or $P_{2}$, then a strong cycle containing $u v$ and $x y$ can be extracted from the parts of the four cycles $P_{1}, P_{2}, Q_{1}$ and $Q_{2}$.

$($ iii $) \Rightarrow(i v)$ Let $\mathrm{x}$ and $\mathrm{y}$ be two nodes and let $(u, v)$ be a strong arc in $G$. Let $x^{\prime}$ be a strong neighbor of $x$ and $y^{\prime}$ be a strong neighbor of $y$. Now there exist a strong cycles $C_{1}$ containing $x x^{\prime}$ and $u v$ and a strong cycle $C_{2}$ containing $y y^{\prime}$ and $u v$. Now $x x^{\prime} \ldots\left(C_{1}\right) \ldots u v \ldots\left(C_{2}\right) \ldots y^{\prime} y$ is a strong $x-y$ path containing the arc $u v$.

$(i v) \Rightarrow(v)$ Let $G$ be a f-block. Let $u, v, w$ be three distinct nodes of $G$. Let $v^{\prime}$ be a strong neighbor of $v$. Then $u$ and $w$ are distinct nodes and $v v^{\prime}$ is a strong arc of $G$. By (iv) there exists a strong path from $u$ to $w$ containing the arc $u v^{\prime}$ (Even if $v^{\prime}=u$ or $w$ ). Thus we have a strong path between the two given nodes containing the third.

$(v) \Rightarrow(v i)$ Let $u, v, w$ be three distinct nodes of $G$. Let $P$ be a strong 
path between $u$ and $w$ containing $v$. Then clearly the $u-v$ strong sub path say $P^{\prime}$ does not contain $w$.

$(v i) \Rightarrow(i)$ Let $u$ and $v$ be two given nodes. Let $w$ be a third node in $G$. Let $P_{1}$ be the strong path joining $u$ and $v$ not containing $w$. Let $P_{2}$ be the strong path joining $u$ and $w$ not containing $v$ and let $P_{3}$ be the strong path joining $v$ and $w$ not containing $u$. Then $P_{1} \bigcup P_{2} \bigcup P_{3}$ will contain a strong cycle containing $u$ and $v$.

Remark 1: The conditions given in Theorem 3 are only necessary, not sufficient for a weighted graph to be a p- block as seen from the following example.

Example 5 : Let $G(V, E)$ be a weighted graph with $V=\{a, b, c, d\}$ and $E=$ $\left\{e_{1}=(a, b), e_{2}=(b, c), e_{3}=(c, d), e_{4}=(d, a)\right\}$ with $w\left(e_{1}\right)=10, w\left(e_{2}\right)=$ $9, w\left(e_{3}\right)=8, w\left(e_{4}\right)=8$.

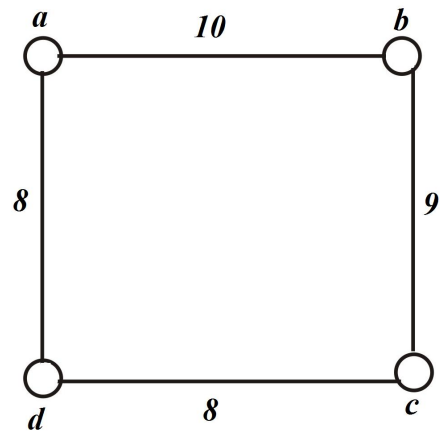

Figure 5 : p-block not satisfying the condition of Theorem.3

In this graph, all arcs are strong. So any two nodes of $G$ lies on a common strong cycle. But $b$ is a partial cutnode. So it is not a p-block.

Definition 9: $A$ cycle $C$ in a weighted graph $G$ is said to be a locamin cycle if there exist a weakest arc of $G$ incident on every node of $G . C$ is called multimim if $C$ has more than one weakest arc of $G$.

Example 6: Let $G(V, E)$ be a weighted graph with $V=\{a, b, c, d\}$ and $E=\left\{e_{1}=(a, b), e_{2}=(b, c), e_{3}=(c, d), e_{4}=(d, a), e_{5}=(a, c)\right\}$ with $w\left(e_{1}\right)=10, w\left(e_{2}\right)=9, w\left(e_{3}\right)=8, w\left(e_{4}\right)=8, w\left(e_{5}\right)=12$. 


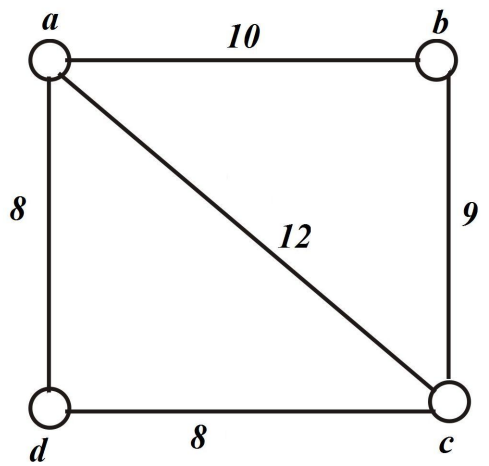

Figure 6: Multimin and locamin cycles

In $G, C_{1}=$ acda is both multimin and locamin. $C_{2}=a b c d a$ is multimin but not locamin. $C_{3}=a b c a$ is neither multimin nor locamin.

Note that a locamin cycle is always multimin . But multimin cycles or locamin cycles need not be strong cycle as seen from the following example.

Example 7 : Let $G(V, E)$ be a weighted graph with $V=\{a, b, c, d, e, f\}$ and $E=\left\{e_{1}=(a, b), e_{2}=(b, c), e_{3}=(c, a), e_{4}=(a, d), e_{5}=(d, b), e_{6}=\right.$ $\left.(b, e), e_{7}=(e, c), e_{8}=(c, f), e_{9}=(a, f)\right\}$ with $w\left(e_{1}\right)=1, w\left(e_{2}\right)=1, w\left(e_{3}\right)=$ $1, w\left(e_{4}\right)=2, w\left(e_{5}\right)=2, w\left(e_{6}\right)=2, w\left(e_{7}\right)=2, w\left(e_{8}\right)=2, w\left(e_{9}\right)=2$.

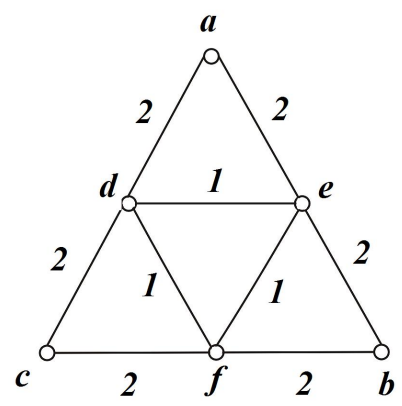

Figure 7 : Multimin and locamin $\delta$ - cycle

In $G, C=a b c a$ is both multimin and locamin, but it contains only $\delta$ arcs. That is $C$ is not a strong cycle. 


\section{Strongest Strong Cycles}

Definition 10: A cycle $C$ in a weighted graph $G$ is called a strongest strong cycle (SSC) if $C$ is the union of two strongest strong $u-v$ paths for every pair of nodes $u$ and $v$ in $C$ except when $(u, v)$ is a p-bridge of $G$ in $C$.

Note that in the above definition, arc $(u, v)$ can be an p-bridge of $G$. But the condition that $C$ is the union of two strongest strong $u-v$ paths can be relaxed only for those nodes which are the end nodes of p-bridges of $G$ which are in $C$. Also, $C O N N_{G}(x, y)=C O N N_{C}(x, y)$ for all nodes $x, y$ in $C$.

Example 8 : Consider the following weighted graph $G(V, E)$ with $V=$ $\{a, b, c, d\}$ and $E=\left\{e_{1}=(a, b), e_{2}=(b, c), e_{3}=(c, d), e_{4}=(d, a), e_{5}=\right.$ $(a, c)\}$ with $w\left(e_{1}\right)=5, w\left(e_{2}\right)=2, w\left(e_{3}\right)=5, w\left(e_{4}\right)=2, w\left(e_{5}\right)=1$.

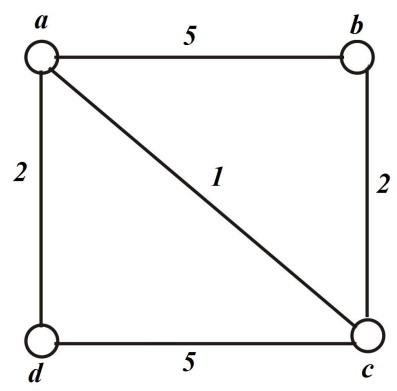

Figure 8 : Strongest strong cycle

In this graph, $C=a b c d a$ is a strongest strong cycle. There are two p-bridges of $G$ in $C$, namely $(a, b)$ and $(c, d)$. We can find two strongest paths joining any other pair of nodes in $C$.

A locamin cycle in a weighted graph $G$ need not be an SSC and an SSC need not be a locamin cycle. But the concepts of locamin cycle and SSC coincides when $G$ is a cycle as seen from the next Theorem.

Theorem 4: Let $G$ be a weighted cycle. Then the following are equivalent.

(i) $G$ is a p-block.

(ii) $G$ is an $S S C$.

(iii) $G$ is a locamin cycle. 
Proof : $(i) \Rightarrow(i i)$

First assume that $G$ is a p-block. Then by theorem 1, there exist two internally disjoint strongest $u-v$ paths for all pairs of nodes $u, v$ in $G$ such that $(u, v)$ is not a p-bridge. Clearly all arcs belonging to these paths are strong; for otherwise if $(x, y)$ is not strong (ie a $\delta$-arc), then $G-(x, y)$ will be the only strongest $x-y$ path in $G$, getting a contradiction.

$($ ii $) \Rightarrow($ iii $)$

Suppose that $G$ is an SSC. If possible suppose that $G$ is not locamin. Then there exists some node $w$ such that $w$ is not on a weakest arc of $G$. Let $(u, w)$ and $(w, v)$ be the two arcs incident on $w$, which are not weakest arcs. This implies that the path $u, w, v$ is the unique strongest $u-v$ path in $G$, contradiction to the assumption that $G$ is an SSC.

$($ iii $) \Rightarrow(i)$

Let $G$ be a locamin cycle. If possible suppose that $G$ has a p-cutnode say $w$. Then for some pair of nodes $u, v$ in $G, C O N N_{G-w}(u, v)<C O N N_{G}(u, v)$. This implies that there exist a unique strongest path between $u$ and $v$ in $G$, which contradicts the assumption that $G$ is an SSC.

Theorem 5: If any two nodes of a weighted graph $G$ lie on common SSC, then $G$ is a p-block.

Proof : Let $G$ be a weighted graph satisfying the condition of the Theorem. Clearly $G$ is connected. Let $w$ be a node in $G$. For any two nodes $x$ and $y$ such that $x \neq w \neq y$, there exists an SSC containing $x$ and $y$. That is there exist two internally disjoint strongest $x-y$ paths in $G$. At most one of these paths can contain the node $w$ and hence $w$ cannot be a p-cutnode of $G$. Since $w$ is arbitrary, it follows that $G$ is a block.

\section{Cycle Connectivity in Weighted Graphs}

In graphs, the strength of each cycle is 1 . But in weighted graphs it is possible that cycles of different strengths pass through different pairs of nodes. In this section, we define two new connectivity concepts in weighted graphs, namely $\theta$ - evaluation and cycle connectivity $C_{u, v}^{G}$. Using these, a new type of weighted graphs called $\theta$ - weighted graphs are defined and p-blocks in $\theta$ - weighted graphs are fully characterized. 
Definition 11: Let $G$ be a weighted graph. Then for any two nodes $u$ and $v$ of $G$, there associated a set say $\theta(u, v)$ called the $\theta$ - evaluation of $u$ and $v$ and is defined as $\theta(u, v)=\{\alpha$ : where $\alpha$ is the strength of a strong cycle passing through both $u$ and $v$.

If there is no strong cycle containing both $u$ and $v$, then define $\theta(u, v)=\phi$.

Definition 12: $\operatorname{Max}\{\alpha: \alpha \in \theta(u, v) ; u, v \in V(G)\}$ is defined as the cycle connectivity between $u$ and $v$ in $G$ and is denoted by $C_{u, v}^{G}$. If $\theta(u, v)=\phi$ for some pair of nodes $u$ and $v$, we define the cycle connectivity between $u$ and $v$ to be 0 .

Example 9 : Let $G(V, E)$ be a weighted graph with $V=\{a, b, c, d\}$ and $E=\left\{e_{1}=(a, b), e_{2}=(b, c), e_{3}=(c, d), e_{4}=(d, a), e_{5}=(a, c)\right\}$ with $w\left(e_{1}\right)=3, w\left(e_{2}\right)=3, w\left(e_{3}\right)=5, w\left(e_{4}\right)=7, w\left(e_{5}\right)=5$.

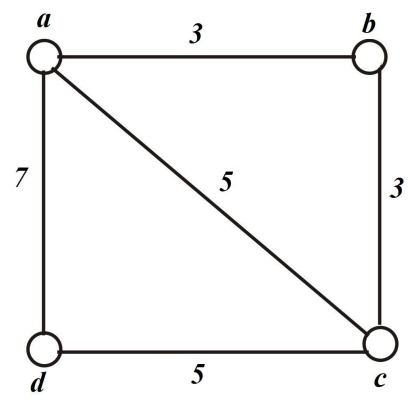

Figure 9 : Cycle connectivity

In this graph, there are three cycles passing through a and c. They are $C_{1}=a b c a, C_{2}=a c d a$ and $C_{3}=a b c d a$. Also $s\left(C_{1}\right)=3, s\left(C_{2}\right)=5$ and $s\left(C_{3}\right)=3$. Here $\theta(a, c)=\{3,5\}$ hence $C_{a, c}^{G}=\max \{3,5\}=5$.

Cycle connectivity is a measure of connectedness of a weighted graph and it is always less than or equal to the strength of connectedness between any two nodes $u$ and $v$. In a graph without weights, the cycle connectivity of any two nodes $u$ and $v$ is 1 if $u$ and $v$ belongs to a common cycle and 0 otherwise. 
Definition 13: Let $G$ be a weighted graph. $G$ is said to be a $\theta$ - weighted graph if $\theta$ - evaluation of each pair of nodes in $G$ is either empty or a singleton set. In other words $G$ is called a $\theta$ - weighted graph if for each pair of nodes $u$ and $v$, either there is no strong cycle passing through $u$ and $v$ or all strong cycles passing through $u$ and $v$ have the same strength.

Example 10 : Let $G(V, E)$ be a weighted graph with $V=\{a, b, c, d\}$ and $E=\left\{e_{1}=(a, b), e_{2}=(b, c), e_{3}=(c, d), e_{4}=(d, a)\right\}$ with $w\left(e_{1}\right)=$ $9, w\left(e_{2}\right)=8, w\left(e_{3}\right)=7, w\left(e_{4}\right)=3$.

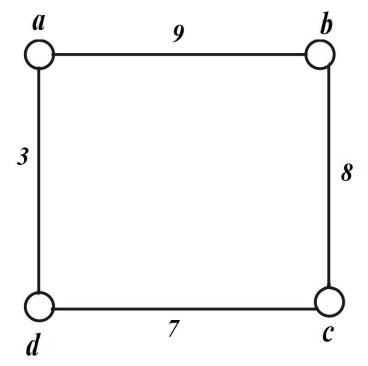

Figure 10 : Trivial $\theta$ - weighted graph

Clearly $G$ is a $\theta$ - weighted graph as $G$ has no strong cycles. Note that in $G$, the arc $e_{4}=(d, a)$ is not strong.

Example 11 : Let $G(V, E)$ be a weighted graph with $V=\{a, b, c, d\}$ and $E=\left\{e_{1}=(a, b), e_{2}=(b, c), e_{3}=(c, d), e_{4}=(d, a), e_{5}=(a, c)\right\}$ with $w\left(e_{1}\right)=3, w\left(e_{2}\right)=2, w\left(e_{3}\right)=2, w\left(e_{4}\right)=5, w\left(e_{5}\right)=2$.

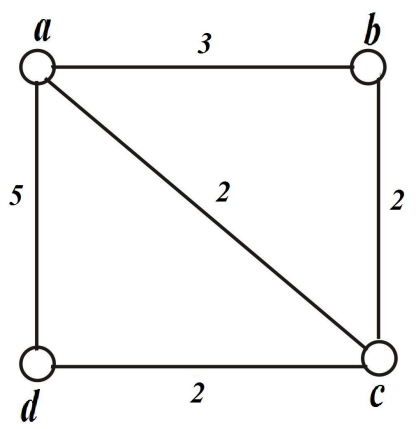

Figure 11: $\theta$ - weighted graph 
In this graph, the $\theta$ - evaluation for any pair of nodes is $\{2\}$ and hence $G$ is a $\theta$-weighted graph.

We now show that in a $\theta$ - weighted graph which is a $p$-block, the concepts of strong path and strongest path coincide and as a result, the concepts of strong cycle and $S S C$ are also equivalent. Thus all the six necessary and sufficient conditions for blocks in graphs can be generalized to p-blocks in weighted graphs

Lemma 1: Let $G$ be a $\theta$ - weighted graph which is a block. Then any strong $u-v$ path such that $(u, v)$ is not a p-bridge is a strongest $u-v$ path and hence any strong cycle in $G$ is a strongest strong cycle.

Proof: Let $G$ be a $\theta$ - weighted graph which is a p-block. Clearly $G$ is connected. Let $u, v \in V(G)$ be such that $(u, v)$ is not a p-bridge. Let $P$ be a strong $u-v$ path in $G$. If $P$ is not a strongest $u-v$ path, then since $G$ is a p-block, there exist two internally disjoint strongest strong $u-v$ paths say $P_{1}$ and $P_{2}$. Then $P_{1} \cup P$ is a strong cycle with strength less than that of the cycle $P_{1} \cup P_{2}$. Both these cycles pass through $u$ and $v$ and hence $\theta(u, v)$ is not a singleton or empty set, which is a contradiction to the fact that $G$ is a $\theta$ - weighted graph. Thus $P$ must be a strongest strong $u-v$ path.

To prove the second assertion of the lemma, let $C$ be a strong cycle in $G$. Let $u, v$ be two nodes in $C$ such that $(u, v)$ is not a p-bridge in $C$. Then by first part both these $u-v$ paths in $C$ are strongest $u-v$ paths. Thus $G$ is a strongest strong cycle.

Theorem 7: Let $G$ be a $\theta$ - weighted graph. Then the following statements are equivalent.

(i) $G$ is a p-block.

(ii) Every two nodes of $G$ lie on a common strongest strong cycle.

(iii) Each node and a strong arc of $G$ lie on a common strongest strong cycle.

(iv) Any two strong arcs of $G$ lie on a common strongest strong cycle.

(v) For any two given nodes $u$ and $v$ such that $(u, v)$ is not a p-bridge and a strong arc $(x, y)$ in $G$, there exists a strongest strong $u-v$ path containing the $\operatorname{arc}(x, y)$. 
(vi) For every three distinct nodes $u_{i}, i=1,2,3$ of $G$ such that $\left(u_{i}, u_{j}\right), j=$ $1,2,3$ and $i \neq j$, is not a p-bridge, there exist strongest strong paths joining any two of them containing the third.

(vii) For every three distinct nodes $u_{i}, i=1,2,3$ of $G$ such that $\left(u_{i}, u_{j}\right), j=$ $1,2,3$ and $i \neq j$, is not a $p$-bridge, there exist strongest strong paths joining any two of them not containing the third.

\section{Proof:}

Theorem 3 and Lemma 1 together give all the required implications except $(v i i) \Rightarrow(i)$.

To prove (vii) $\Rightarrow(i)$, note that for any node $w$ of $G$ and for every pair of nodes $x, y$ other than $w$, there exists a strongest $x-y$ path not containing the node $w$. Clearly $G$ is connected. Thus node $w$ is not in every strongest $x-y$ path for all pair of nodes $x$ and $y$ and hence $w$ is not a p-cutnode. Since $w$ is arbitrary, it follows that $G$ is a p-block.

\section{Concluding remarks}

Weighted graphs are the precise models of all kinds of networks. Connectivity is the most important concept in the entire graph theory. But in classical problems connectivity concepts deals with the disconnection of the networks. The reduction in flow is more frequent than the disconnection. The authors made an attempt to introduce partial cutnodes, bridges and blocks dealing with the reduction in the strength of connectedness between different pairs of nodes in a weighted graph. Also an attempt is made to characterize partial blocks in weighted graphs using different types of cycles. It is fully characterized in the case of a particular subclass of weighted graphs.

\section{References}

[1] J. A. Bondy, G. Fan, Optimal paths and cycles in weighted graphs, Ann. Discrete Mathematics 41, pp. 53-69, (1989).

[2] J. A. Bondy, G. Fan, Cycles in weighted graphs, Combinatorica 11, pp. 191-205, (1991). 
[3] R. Diestel, Graph Theory, Second edition, Graduate texts in mathematics 173, Springer, (2000).

[4] G. A. Dirac, Some theorems on abstract graphs, Proc. London Math. Soc. (3) 2, pp. 69 - 81, (1952).

[5] M. Grotschel, Graphs with cycles containing given paths, Ann. Discrete Math. 1, pp. 233 - 245, (1977).

[6] Sunil Mathew, M. S. Sunitha, Types of arcs in a fuzzy graph, Information Sciences 179 (11)1, pp. 1760-1768, (2009).

[7] Sunil Mathew, M. S. Sunitha, Some connectivity concepts in weighted graphs, Advances and Applications in Discrete Mathematics 6 (1), pp. 45-54, (2010).

[8] Sunil Mathew, M. S. Sunitha, Bonds in graphs and fuzzy graphs,Advances in Fuzzy Sets and Systems, 6 (2), pp. 107-119, (2010).

[9] S. Zang, X. Li, H. Broersma, Heavy paths and cycles in weighted graphs, Discrete Math. 223, pp. 327-336, (2000).

\section{Sunil Mathew}

Department of Mathematics

National Institute of Technology

Calicut - 673601

India

e-mail : sm@nitc.ac.in

and

\section{S. Sunitha}

Department of Mathematics, National Institute of Technology

Calicut - 673601

India

e-mail : sunitha@nitc.ac.in 